\title{
Exploration of Nano-scale Structural Instabilities in Metastable $\beta$ Titanium Alloys Using Advanced Electron Microscopy
}

\author{
Yufeng Zheng ${ }^{1,2, *}$, Dong Wang ${ }^{3}$, Rajarshi Banerjee ${ }^{4}$, Dipankar Banerjee ${ }^{5}$, Yunzhi Wang ${ }^{1}$, Hamish L. \\ Fraser $^{1}$
}

${ }^{1}$ Center for the Accelerated Maturation of Materials (CAMM) and Department of Materials Science and Engineering, The Ohio State University, 1305 Kinnear Road, Columbus, OH 43212, USA.

${ }^{2}$ Department of Chemical and Materials Engineering, University of Nevada Reno, 1664 N. Virginia St., Reno, NV 89557, USA

${ }^{3}$ Center of Microstructure Science, Frontier Institute of Science and Technology, State Key Laboratory for Mechanical Behavior of Materials, Xi'an Jiaotong University, Xi'an, Shaanxi, China

${ }^{4}$ Materials Research Facility and Department of Materials Science and Engineering, University of North Texas, Denton, TX 76207, USA

${ }^{5}$ Department of Materials Engineering, Indian Institute of Science, Bengaluru, Karnataka 560012, India

* Corresponding author: Yufeng Zheng, yufengz@unr.edu, Department of Chemical and Materials Engineering, University of Nevada Reno, 1664 N. Virginia St., Reno, NV 89557, USA

Keywords: Microstructure evolution; Metastable $\beta$ titanium alloys; $\omega$ phase; O' phase; Incommensurate modulated domains

\begin{abstract}
$\underline{\text { Abstract }}$
A variety of nano-scale structural instabilities formed in different metastable $\beta$ titanium alloys have been systematically investigated using advanced characterization techniques. The characteristics of three different types of nano-scale structural instabilities, the transformation mechanisms and pathways involved and the critical experimental conditions to generate such nano-scale phases will be reviewed and summarized, including athermal $\omega$ phase with hexagonal structure, $\mathrm{O}^{\prime}$ phase with orthorhombic structure, and incommensurate modulated nanodomains. The athermal $\omega$ phase has been observed in the as-quenched state in Ti-xMo ( $\mathrm{x}=12,15$ and 18 ${ }^{1}$ ), Ti-18Mo-5Al, Ti-20V, Ti-5Fe, Ti-5Al-5Mo-5V-3Cr (Ti-5553) and Ti-24Nb4Zr-8Sn (Ti-2448). O' phase has been characterized to co-exist with athermal $\omega$ phase in the as-quenched state isomorphous titanium alloys, including Ti-26Zr-2Al (at.\%), Ti-18Mo, Ti-18Mo-5Al, Ti-5553 and Ti2448. Incommensurate modulated nanodomains were found in compositionally graded Ti-xFe alloy when the athermal $\omega$ phase is suppressed. These various nano-scale structural instabilities need to be taken into consideration when designing novel metastable $\beta$ titanium alloys to optimize the mechanical performance by microstructure engineering.
\end{abstract}

\section{Introduction}

Metastable $\beta$ titanium alloys have attracted significant amount of attention in recent years, due to the combination of great properties, such as high strength, low modulus, excellent corrosion resistance and superior creep resistance at intermediate temperature $[1,2]$. The microstructure of metastable $\beta$ titanium alloys is very sensitive to the composition and thermo-mechanical processes, and therefore its performance can be manipulated by the accurately controlled microstructure engineering [3]. In the last decade, the design of novel metastable $\beta$ titanium alloys mainly arises from two strategies: To improve the strength by the precipitation strengthening involving refined scale $h c p$ structure alpha phase [4] and to improve the ductility by the introduction of TRIP (transformation induced plasticity) and TWIP (twinning induced plasticity) effect [5]. Both of these two strategies focus on the very narrow composition range in which martensite ( $\alpha^{\prime}$ or $\left.\alpha^{\prime \prime}\right)$ phase is suppressed at room temperature, but many other nano-scale structural instabilities may also be formed [6]. With the development of the advanced characterization techniques, especially the atomic resolution aberration-corrected scanning transmission electron microscopy, a variety of nano-scale novel phases have been reported by different research groups in metastable $\beta$ titanium alloys: A new B2 structured

${ }^{1}$ All compositions introduced in this manuscript are in weight percent if not specified individually. 
phase was characterized in the thin foil heat treated at $300^{\circ} \mathrm{C}$ via both in-situ and ex-situ TEM observations in Ti-15Mo [7]. Nanoscale diffusionless isothermal $\omega$ phase was reported for the first time in Ti-V alloys driven by the compositional fluctuation [8]. In the solution-and-quenched metastable $\beta$ titanium alloys, Kim et. al. reported the nano-scale domains formed in Ti-Nb-O alloys due to the atom shuffle activated by the local strain field of interstitial oxygen atom [9]. Wang et. al. reported nano-scale martensite formation by strain glass transition in gum metal [10]. What is more, $\delta$ phase was reported in the gum-like Ti-2448 using high energy synchrotron XRD [11]. In our earlier work, various nano-scale structural and compositional instabilities have been explored. Three different types of $\omega$ phase have been explored including athermal $\omega$ phase in the as-quenched state Ti-xMo ( $\mathrm{x}=12,15$ and 18) [12], Ti-18Mo-5Al, Ti-20V [13], Ti-5Fe, Ti-5553 [14] and Ti-2448 [15], isothermal $\omega$ phase in the intermediate temperature aged Ti-xMo $(x=12,15$ and 18), Ti-18Mo-5Al [16], Ti-20V [17] and Ti-5553 [18] and stress-induced $\omega$ phase in deformed Ti-2448 [19]. Disordered orthorhombic structure O' phase was observed in Ti-26Zr-2Al (at.\%) [20], Ti-18Mo [12], Ti$18 \mathrm{Mo}-5 \mathrm{Al}$ [21], Ti-5553 [22] and Ti-2448 [15]. Ordered orthorhombic structured phase was observed in the aged Ti-5553 using both diffraction contrast TEM and Z-contrast HAADF-STEM techniques [22, 23]. Incommensurate modulated nanodomains were found in compositionally graded Ti-xFe alloy [24].

In this paper, the results from studies of nano-scale precipitation in metastable $\beta$ titanium alloys is presented, focusing on three types of nano-scale phases formed by atom shuffles during fast quenching. In the first part of this work, nano-scale instabilities, athermal $\omega$ phase, $\mathrm{O}^{\prime}$ phase in isomorphous type titanium alloys and incommensurate modulated nanodomains in eutectoid type titanium alloys are discussed. The structure of these nano-scale instabilities has been investigated using aberration-corrected S/TEM to determine their formation mechanisms. The second part of the work is focused on the comparison between nano-scale phases present in the isomorphous titanium alloys and those in eutectoid titanium alloys.

\section{Materials and experiments}

The microstructure in a number of different metastable $\beta$ titanium alloys was investigated in this work using advanced electron microscopy. Alloy samples of Ti-12Mo, Ti-15Mo, Ti-18Mo, Ti-18Mo-5Al and Ti-5553 were provided by TIMET Company; Ti-2448 was provided by the Institute of Metal Research, Chinese Academy of Science; Ti-20V, compositionally graded Ti-Fe, Ti-26Nb and Ti-26Nb-2Zr were prepared in the laboratory via arc-melting system. All the samples studied were solutionized in the single $\beta$ phase temperature range using conventional tube furnace or electro-thermal-mechanical-tester under vacuum followed by fast cooling to room temperature. The heat-treated samples were prepared for scanning electron microscopy (SEM) analysis using conventional grinding and polishing routines. Thin foils for transmission electron microscopy (TEM) characterization were prepared from the center of the polished samples, using Dual-Beam Focused-Ion-Beam FEI Helios and Nova Nanolab systems, and finally cleaned using Fischione Model 1040 Nanomill. Diffraction contrast TEM analysis was conducted on Philips CM200 microscope at 200kV or FEI Tecnai30 microscope at $300 \mathrm{kV}$. Z-contrast aberration-corrected high angle annular dark field- scanning transmission electron microscopy (HAADF-STEM) imaging was performed on probe-corrected FEI Titan $80-300$ at $300 \mathrm{kV}$.

\section{Results}

In all nine different metastable $\beta$ titanium alloys studied in this work, athermal $\omega$ phase has been observed in the as-quenched state. One example from Ti-15Mo is shown in Fig. 1, recorded from the $\beta$ solutionized $\left(1000^{\circ} \mathrm{C}\right.$ for $\left.30 \mathrm{mins}\right)$ and water quenched sample. In the inset $[110]_{\beta}$ zone axis selected area diffraction (SAD) pattern, clear $\omega$ reflections at $1 / 3$ and $2 / 3$ of $\{112\}_{\beta}$ are observed. Correspondingly, the dark field image obtained from the $\omega$ phase reflections marked by the yellow color circle in Fig. 1(a) shows a large number density of nano-scale athermal $\omega$ phase particles evenly distributed in the parent $\beta$ phase matrix. The structure of one of these athermal $\omega$ phase particle is shown in Fig. 1(b) where the incomplete collapse of $\{111\}_{\beta}$ atom planes can be clearly seen. It is consistent with the observation in Ref. [12] that the solute content of Mo governs the degree of plane collapse in athermal $\omega$ phase. The orientation relationship between $\omega$ phase and $\beta$ phase observed is: $(0001)_{\omega} / /(111)_{\beta}$ and $[11-20]_{\omega} / /[1-10]_{\beta}$. 

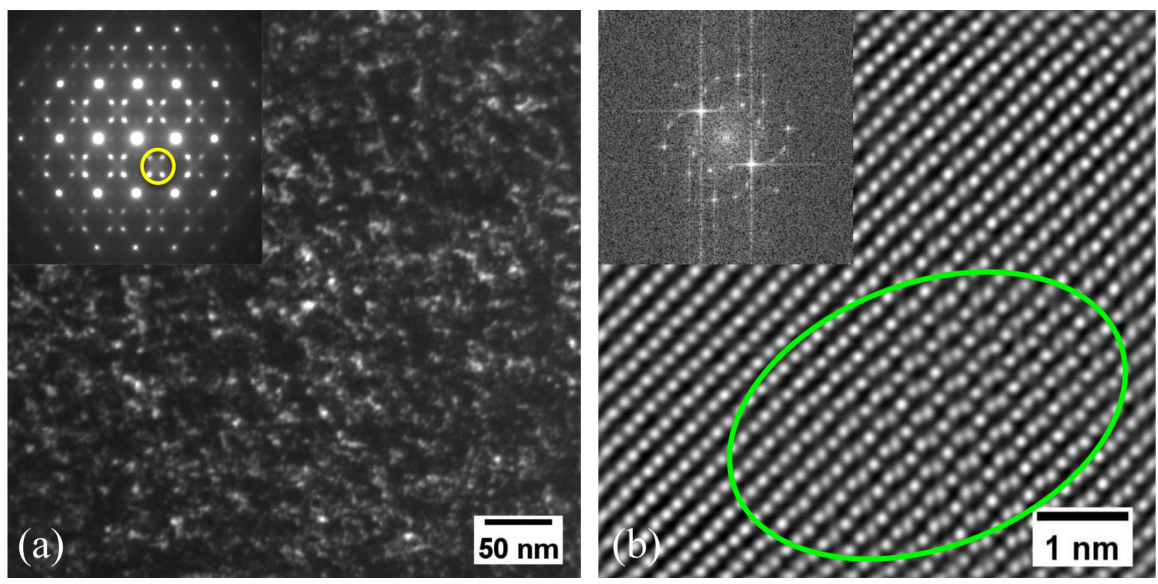

Figure 1. Athermal $\omega$ phase in as-quenched Ti-15Mo: (a) TEM SAD pattern and corresponding dark field image showing athermal $\omega$ phase; (b) Z-contrast HAADF-STEM image and corresponding fast Fourier transformation (FFT) showing the structure of athermal $\omega$ phase.

$\mathrm{O}^{\prime}$ phase with the disordered orthorhombic structure is observed in Ti-18Mo, Ti-18Mo-5Al, Ti-26Nb-2Zr (at\%), Ti-5553 and Ti-2448, all in the as-quenched state. One example is shown in Fig. 2 from as-quenched Ti-18Mo-5Al. In the inset, the $[110]_{\beta}$ zone axis selective area diffraction pattern is shown with clear $\mathrm{O}^{\prime}$ reflections at $1 / 2$ of $\{112\} \beta$. A large number density of nano-scale $\mathrm{O}^{\prime}$ phase are present in the parent $\beta$ phase matrix together with $\omega$ phase particles in the dark field image obtained from selected reflections marked by yellow color circle in Fig. 2(a). The structure of several O' phase particles is shown in Fig. 2(b) where a small degree of atom shuffle of every other $\{110\}_{\beta}$ planes can be observed. The orientation relationship between $\mathrm{O}^{\prime}$ phase and $\beta$ phase observed is: $(001)^{\prime} / /(011)_{\beta}$ and $[100]_{\mathrm{o}^{\prime}} / /[100]_{\beta}$.

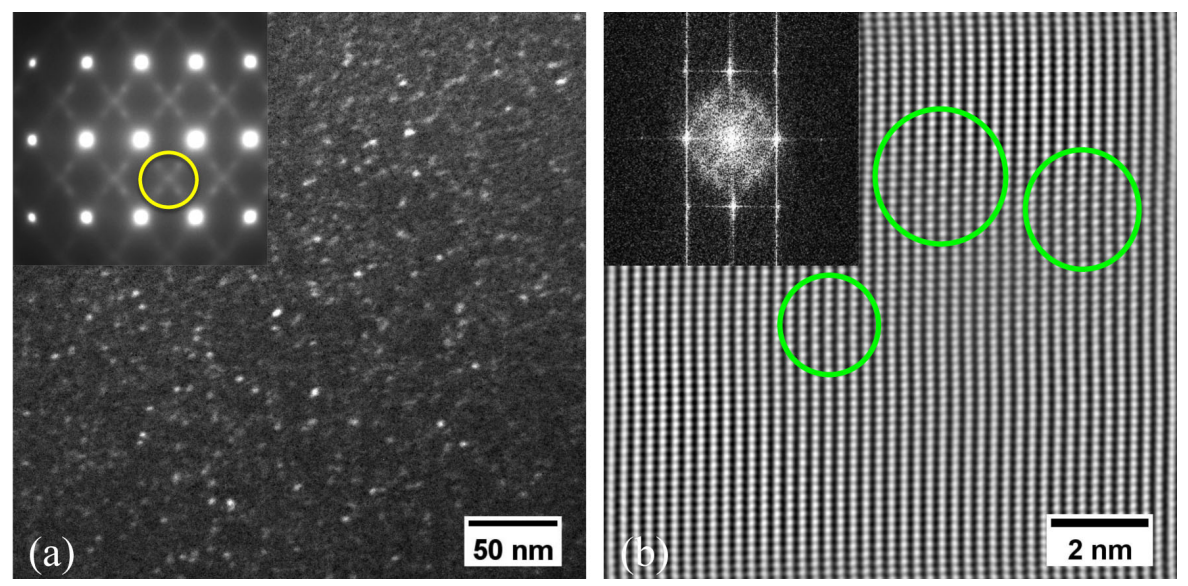

Figure 2. O' phase in as-quenched Ti-18Mo-5Al: (a) TEM SAD pattern and corresponding dark field image showing $\mathrm{O}^{\prime}$ phase; (b) Z-contrast HAADF-STEM image and corresponding FFT showing the structure of O' phase.

The following conclusions regarding $\mathrm{O}^{\prime}$ phase can be summarized:

(1) O' phase, having a disordered orthorhombic structure, is a nano-scale metastable phase formed in isomorphous type $\beta$ titanium alloy by the shuffling of atoms (shuffling in the $<01 \overline{1}>$ directions on every other $\{011\}$ plane,) when the total solute content of the alloy exceeds a critical value.

(2) The formation of the $\mathrm{O}^{\prime}$ phase is sensitive to the solute content, and can be accelerated by the addition of both substitutional solute, like $\mathrm{Zr}$ into $\mathrm{Ti}-\mathrm{Nb}$ alloy and $\mathrm{Al}$ into Ti-Mo alloy, and interstitial solute, like oxygen into $\mathrm{Ti}-\mathrm{Nb}$ alloy, but such solute additions of different elements, such as aluminum and oxygen, is not necessary.

Incommensurate modulated domains were observed in the as-quenched state of compositionally-graded TiFe sample, in the region containing an Fe content of between $13.8 \%$ and 33.9\% [24]. The diffraction pattern and high resolution image in Fig. 3 show an example of the domains in the region near B2/BCC interface. In 
the $[110]_{\beta}$ zone axis SAD pattern shown in Fig. 3(a), additional reflections are seen shifted from their regular $1 / 3$ and $2 / 3\{112\}_{\beta}$ positions consistent with the incommensurate modulated domains. The structures of several incommensurate modulated domains are shown in Fig. 3(b), where atom columns can be observed deviating from the $b c c$ lattice sites without a fixed periodicity.
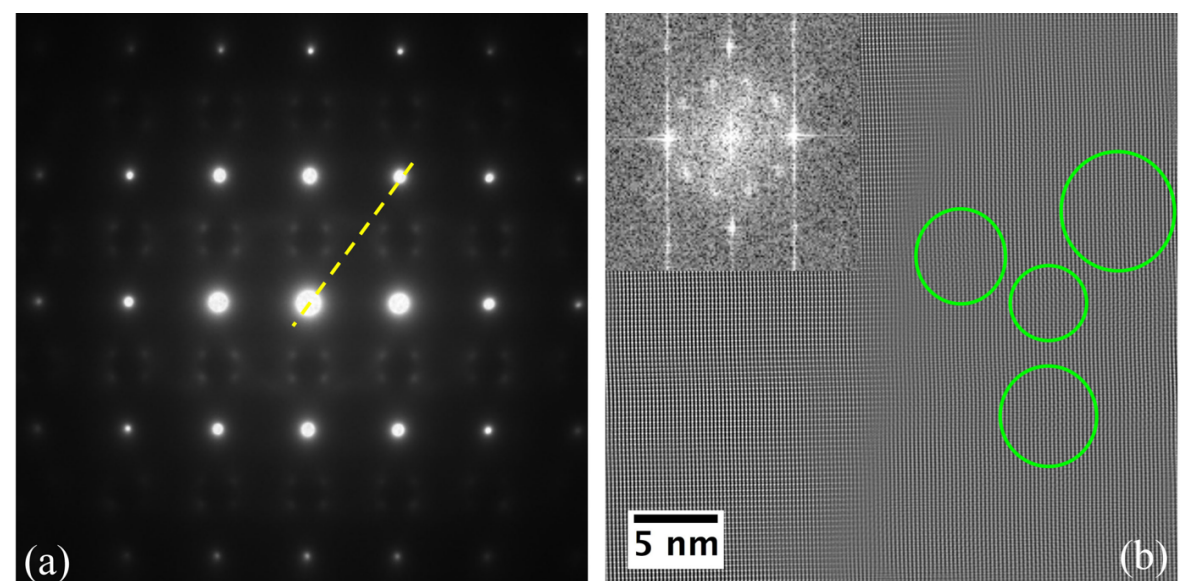

Figure 3. Incommensurate modulated nano-domains in the compositionally graded Ti-Fe sample: (a) TEM SAD pattern showing incommensurate modulated nano-domains; (b) Z-contrast HAADF-STEM image and corresponding FFT showing incommensurate modulated nano-domains.

\section{Discussion}

As has been shown in the above section, experimental characterization from a variety of metastable $\beta$ titanium alloys clearly indicate that with the increase of solute content in metastable $\beta$ titanium alloys, including Ti12Mo, Ti-15Mo, Ti-18Mo, Ti-18Mo-5Al, Ti-26Nb (at\%), Ti-26Nb-2Zr (at\%), Ti-5553 and Ti2448, athermal $\omega$ phase can form in the as-quenched condition by a diffusionless, atom shuffle mechanism. Every two of three adjacent $\{111\}_{\beta}$ atom planes will shuffle towards each other while the third one is unaltered. In isomorphous titanium alloys, such as Ti-18Mo, Ti-18Mo-5Al, Ti-26Nb-2Zr (at\%), Ti-5553 and Ti-2448, the $\mathrm{O}^{\prime}$ phase with the orthorhombic structure can form together with the athermal $\omega$ phase during quenching, by the atom shuffling of every other $\{110\}_{\beta}$ planes along the $\langle 1-10\rangle_{\beta}$ direction, as shown in Fig. 4(a). In eutectoid titanium alloys, such as Ti-Fe alloy, incommensurate modulated domains form by the mechanism of atom shuffle, shown in Fig. 4(b), when the precipitation of the athermal $\omega$ phase is suppressed due to the significant amount of solute addition. Atoms in the incommensurate modulated domains randomly deviated from the $b c c$ lattice but do not exhibit any fixed long-range periodicity.
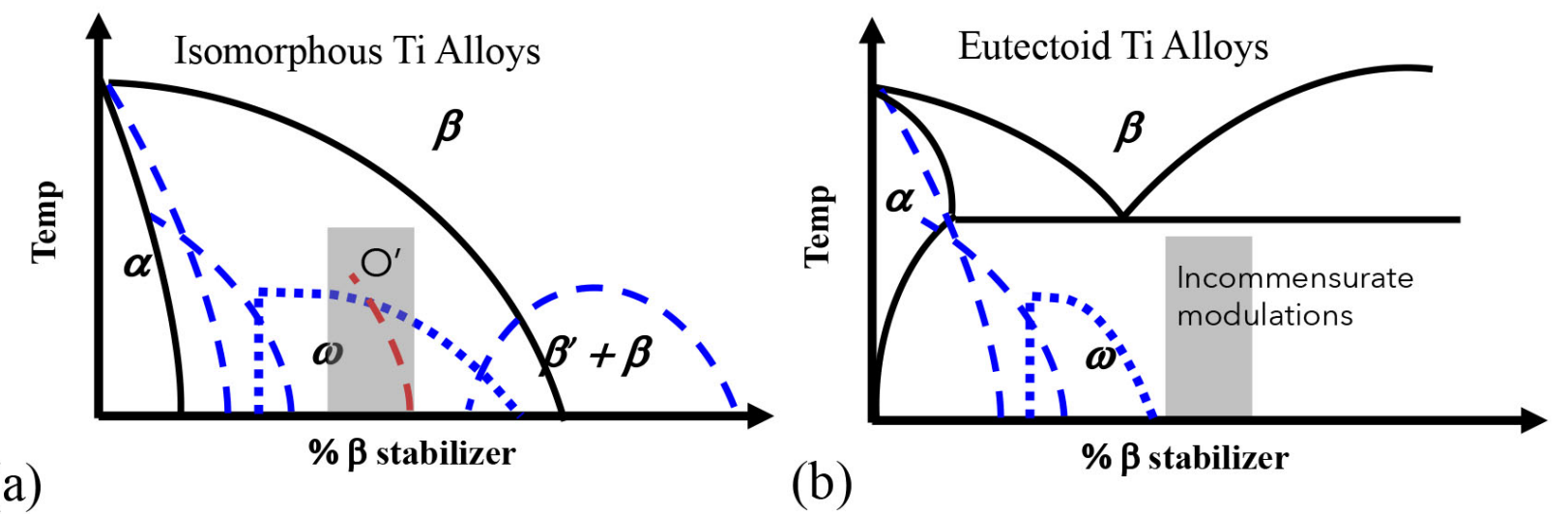

Figure 4. Schematic drawing of pseudo-binary phase diagram for (a) isomorphous Ti alloys and (b) eutectoid Ti alloys

\section{Conclusion}

In this work, three types of nano-scale structural instabilities in isomorphous and eutectoid titanium alloys have been systematically studied using advanced characterization techniques, showing that the solute concentration (type and amount) plays a critical role in determining the nature of nano-scale structural 
instability. Athermal $\omega$ phase can form in both isomorphous and eutectoid titanium alloys; $\mathrm{O}$ ' phase is formed only in isomorphous titanium alloys and can co-exist with athermal $\omega$ phase; nanoscaled incommensurate modulated domains are observed in eutectoid titanium alloys when the athermal $\omega$ phase is suppressed during quenching.

\section{Acknowledgements}

This research has been supported by the National Science Foundation, Division of Materials Research, grant \# DMR-1309270 and DMREF grant DMR-1435483.

\section{References:}

[1] D. Banerjee, J.C. Williams, Acta Materialia 61(2013) 844-879.

[2] R.P. Kolli, A. Devaraj, Metals 8(2018) 506.

[3] J.D. Cotton, R.D. Briggs, R.R. Boyer, S. Tamirisakandala, P. Russo, N. Shchetnikov, J.C. Fanning, JOM 67(2015) 1281-1303.

[4] A. Devaraj, V.V. Joshi, A. Srivastava, S. Manandhar, V. Moxson, V.A. Duz, C. Lavender, Nature Communications 7 (2016) 11176.

[5] F. Sun, J.Y. Zhang, M. Marteleur, C. Brozek, E.F. Rauch, M. Veron, P. Vermaut, P.J. Jacques, F. Prima, Scripta Materialia 94 (2015) 17-20.

[6] M. Abdel-Hady, K. Hinoshita, M. Morinaga, Scripta Materialia 55 (2006) 477-480.

[7] J.M. Bennett, E.J. Pickering, J.S. Barnard, D. Rugg, H.J. Stone, N.G. Jones, Materials Characterization 142 (2018) 523-530.

[8] M. Tane, H. Nishiyama, A. Umeda, N.L. Okamoto, K. Inoue, M. Luckabauer, Y. Nagai, T. Sekino, T. Nakano, T. Ichitsubo, Physical Review Materials 3(2019) 043604.

[9] H.Y. Kim, L. Wei, S. Kobayashi, M. Tahara, S. Miyazaki, Acta Materialia 61 (2013) 4874-4886.

[10] Y. Wang, J. Gao, H. Wu, S. Yang, X. Ding, D. Wang, X. Ren, Y. Wang, X. Song, J. Gao, Scientific Reports 4 (2014) 3995.

[11] J.P. Liu, Y.D. Wang, Y.L. Hao, Y. Wang, Z.H. Nie, D. Wang, Y. Ren, Z.P. Lu, J. Wang, H. Wang, Scientific reports 3 (2013) 2156.

[12] Y. Zheng, D. Banerjee, H.L. Fraser, Scripta Materialia 116 (2016) 131-134.

[13] D. Choudhuri, Y. Zheng, T. Alam, R. Shi, M. Hendrickson, S. Banerjee, Y. Wang, S.G. Srinivasan, H. Fraser, R. Banerjee, Acta Materialia 130 (2017) 215-228.

[14] Y. Zheng, R.E.A. Williams, J.M. Sosa, Y. Wang, R. Banerjee, H.L. Fraser, Scripta Materialia 111 (2016) 81-84.

[15] Q. Liang, Y. Zheng, D. Wang, Y. Hao, R. Yang, Y. Wang, H.L. Fraser, Scripta Materialia 158 (2019) 95-99.

[16] Y. Zheng, T. Alam, R.E.A. Williams, S. Nag, R. Banerjee, H.L. Fraser, Structural and Compositional Characteristics of Isothermal Omega Phase in Beta Titanium Alloys, Proceedings of The 13th World Conference on Titanium, Wiley, San Diego, 2015, 559-564.

[17] Y. Zheng, D. Choudhuri, T. Alam, R.E. Williams, R. Banerjee, H.L. Fraser, Scripta Materialia 123 (2016) 81-85.

[18] Y. Zheng, R.E.A. Williams, D. Wang, R. Shi, S. Nag, P. Kami, J.M. Sosa, R. Banerjee, Y. Wang, H.L. Fraser, Acta Materialia 103 (2016) 850-858.

[19] Q. Liang, Z. Kloenne, Y. Zheng, D. Wang, S. Antonov, Y. Gao, Y. Hao, R. Yang, Y. Wang, H.L. Fraser, Scripta Materialia 177 (2020) 181-185.

[20] Y. Zheng, R.E. Williams, S. Nag, R. Banerjee, H.L. Fraser, D. Banerjee, Scripta Materialia 116 (2016) 49-52.

[21] Y. Zheng, T. Alam, R. Banerjee, D. Banerjee, H.L. Fraser, Scripta Materialia 152 (2018) 150-153.

[22] Y. Zheng, S. Antonov, Q. Feng, R. Banerjee, D. Banerjee, H.L. Fraser, Scripta Materialia 176 (2020) 711.

[23] Y. Zheng, R.E.A. Williams, H.L. Fraser, Scripta Materialia 113 (2016) 202-205.

[24] Y. Zheng, D. Huber, H.L. Fraser, Scripta Materialia 154 (2018) 220-224. 